UCRL-JC-128004

PREPRINT

\title{
Improved Gas-filled Hohlraum Performance on Nova with Beam Smoothing
}

\author{
R. L. Kauffman, L. V. Powers, S. N. Dixit, S. G. Glendinning, \\ S. H. Glenzer, R. K. Kirkwood, O. L. Landen, B. J. MacGowan, \\ J. D. Moody, T. J. Orzechowski, D. M. Pennington, G. F. Stone, \\ L. J. Suter, R. E. Turner, T. L. Weiland, A. L. Richard, M. A. Blain
}

This paper was prepared for submittal to the 39th Annual Meeting of the American Physical Society

Division of Plasma Physics

Pittsburgh, PA

November 17-21, 1997

December 2, 1997

This is a preprint of a paperintended for publication in a journal or proceedings. Since changes may be made before publication, this preprint is made available with the understanding that it will not be cited or reproduced without the permission of the author. 


\section{DISCLAIMER}

This document was prepared as an account of work sponsored by an agency of the United States Government. Neither the United States Government nor the University of California nor any of their employees, makes any warranty, express or implied, or assumes any legal liability or responsibility for the accuracy, completeness, or usefulness of any information, apparatus, product, or process

disclosed, or represents that its use would not infringe privately owned rights. Reference herein to any specific commercial product, process, or service by trade name, trademark, manufacturer, or otherwise, does not necessarily constitute or imply its endorsement, recommendation, or favoring by the United States Government or the University of California. The views and opinions of authors expressed herein do not necessarily state or reflect those of the United States Government or the University of California, and shall not be used for advertising or product endorsement purposes. 


\title{
Improved Gas-filled Hohlraum Performance on Nova with Beam Smoothing*
}

Robert L. Kauffman, L. V. Powers, S. N. Dixit, S. G. Glendinning, S. H.

Glenzer, R. K. Kirkwood, O. L. Landen, B. J. MacGowan, J. D. Moody, T. J. Orzechowski, D. M. Pennington, G. F. Stone, L. J. Suter, R. E. Turner, and T. L. Weiland

Lawrence Livermore National Laboratory, University of California, Livermore, CA 94551

A. L. Richard and M. A. Blain

Commissariat à l'Energie Atomique, Centre d'Études de LimeilValenton, B.P. n. 27, 94190, Villeneuve-Saint-Georges, FR

\begin{abstract}
Gas-filled hohlraums are presently the base line ignition target design for the National Ignition Facility. Initial Nova experiments on gasfilled hohlraums showed that radiation temperature was reduced due to SBS and SRS scattering losses and that implosion symmetry had shifted compared with vacuum hohlraums and calculations. Subsequent single beam experiments imaging thermal $x$-ray emission showed the shift is due to laser-plasma heating dynamics and filamentation in a flowing plasma. Experiments using a single beam have shown that scattering losses and effects of filamentation are reduced when the beam is smoothed with an random phase plate (RPP) or kinoform phase plate (KPP). Scattering is further reduced to less than $5 \%$ of the incident laser energy when SSD is added.
\end{abstract}




\section{Introduction}

For indirect drive ICF, high-Z hohlraums convert laser light into $x$ rays which symmetrically compress and heat the fuel capsule.[1] Laser light heats the high- $Z$ walls producing $x$ rays which then heat the unirradiated walls producing a nearly uniform $x$-ray drive. Hohlraums effectively smooth all but the lowest order implosion symmetry modes. Sources of drive asymmetry are primarily hot spot emission from the laser beams irradiating the walls and absence of emission from the laser entrance holes (LEH's). These asymmetries are time-dependent due to changing albedo of the $x$-ray heated walls and movement of the hot spots due to wall expansion. To successfully ignite and burn capsules on the National Ignition Facility (NIF), hohlraums must efficiently convert laser light to $x$-ray drive and time-integrated drive symmetry on the capsule must be controlled to $\sim 1 \%$.[2]

Ignition target designs for NIF use hohlraums filled with low-Z gas to control the beam hot spot motion.[2] In a vacuum hohlraum the ablated high- $Z$ plasma expands filling the hohlraum volume. For large hohlraums and long pulses such as those required for ignition, the high- $Z$ plasma can exceed several times the inverse bremstrrahlung absorption length of the laser light. Laser deposition can move significantly away from the hohlraum wall producing large changes in time dependent symmetry which are difficult to control accurately. To reduce hohlraum filling with high- $Z$ plasma, ignition hohlraums are filled initially with low- $Z$ gas. The gas when ionized fills the hohlraum with low- $Z$ plasma reducing the amount of high- $Z$ plasma filling the hohlraum. The laser light can propagate through the low- $\mathrm{Z}$ plasma with low absorption and deposit its energy in the high density, high- $Z$ wall. 
The physics of indirect drive hohlraums have been extensively investigated using the Nova laser.[3] Vacuum hohlraums experiments have shown laser light is converted to $x$-ray drive with efficiencies greater than $70 \% .[4,5]$ Asymmetry from the lowest order Legendre mode (P2) is controlled by optimizing beam pointing on the hohlraum wall which controls the hot spot emission relative to the LEH and wall albedo. Implosion symmetry experiments have demonstrated that P2 symmetry can be predicted and measured to $\sim 1 \%$ using vacuum and low-Z-lined hohlraums.[6,7]

Experiments on Nova have tested gas-filled hohlraum performance. In these experiments, whose conditions are described below, the plasma conditions are chosen to approximate part of the NIF hohlraum conditions. At the peak of the laser pulse, the plasma conditions near the LEH are $\sim 3 \mathrm{keV}$ at electron densities $\sim 0.7-1 \times 10^{21} \mathrm{~cm}^{-3}$, comparable to NIF conditions near the LEH. Also the density, density scalelength, and $T_{e}$ in the high- $Z$ plasma near the gas- $A u$ interface are similar. The scalelengths in the underdense plasma is less in these Nova experiments compared to NIF targets because the sizes are smaller. Scattering levels in underdense plasmas have been investigated in separate experiments.[8,9] With unsmoothed Nova beams $x$-ray drive is reduced compared with vacuum hohlraums using Nova beams without any smoothing. As much as $15-20 \%$ of the incident laser light is scattered from the hohlraum causing the reduced drive.[10] In symmetry experiments with $\mathrm{CH}_{4}$ filled hohlraums, the symmetry dependence as a function of pointing is similar to that predicted by simulations, but the predicted pointing for best symmetry differs by $\sim 130 \mu \mathrm{m}$ compared to experiment.[11] 
These experiments, as well as some of the imaging experiments described below, have allowed a physical model to be developed for gas-filled hohlraums. The light scattering from stimulated Brillouin scattering (SBS) and stimulated Raman scattering (SBS) occur in two distinct regions. From the SBS spectrum and correlations with simulations, SBS appears to be produced at densities of $\sim 0.1 \mathrm{n}_{\mathrm{c}}$ or greater. These densities are in the $\mathrm{Au}$ plasma near the gas-Au interface. From the SRS spectrum, SRS is occurring in the underdense plasma at densities less than $0.1 n_{c}$. The spectrum extends to much shorter wavelengths corresponding to much lower densities consistent with SRS being produced in filaments in the low density plasma. The shift in implosion symmetry is also consistent with filamentation near the LEH where the beam propagates transversely through the sonic point. When filamentation occurs in a plasma with transverse flow, the density depression associated with the filament is carried downstream and refracts the beam in the direction of the flow.[12] Simulations show that this effect can cause significant deflection of hot spots for the calculated plasma parameters in these gas-filled hohlraums where the flow perpendicular to the beam is approximately equal to the sound speed.[13] Calculations indicate that spatial and temporal beam smoothing can improve energy coupling and reduce beam deflection.

This paper summarizes the results from smoothed beam experiments on gasfilled hohlraums performed on the Nova laser. The experiment is briefly described in Section II. In Section III, results of the thermal x-ray imaging experiments are presented. These results corroborate the model of beam deflection due to filamentation in a plasma with transverse flow. In Section IV, drive experiments with ten smoothed beams are presented. These 
show that the drive is increased by $\sim 15 \mathrm{eV}$ with beam smoothing approaching levels measured in vacuum hohlraums. The increase in drive is correlated with increased absorption. In symmetry experiments reported elsewhere, the shift in measured pointing for best symmetry is much closer to the predicted pointing of best symmetry.[14] The results are summarized in Section V.

\section{Experiment}

We use the Nova laser to irradiate $\mathrm{CH}_{4}$-filled and vacuum $\mathrm{Au}$ hohlraums (typically, $1600 \mu \mathrm{m}$ diameter $\times 2550 \mu \mathrm{m}$ long). Drive experiments are done in a geometry similar to that in ref. 4 . For these experiments only the $x$-ray diode (XRD) measurements of the reradiated flux are made.[15] The $\mathrm{CH}_{4}$-filled hohlraums had the laser entrance holes and viewing hole for the XRD's covered with $0.35 \mu \mathrm{m}$ polyimide to retain the gas. The vacuum hohlraums also had $0.35 \mu \mathrm{m}$ polyimide covering the XRD diagnostic hole for comparing with the $\mathrm{CH}_{4}$-filled hohlraums. Control experiments showed that the polyimide had negligible effect on the XRD signals. All of the $\mathrm{CH}_{4}$-filled hohlraums were filled to $1 \mathrm{~atm}$. which was monitored until just before the shot.

The laser pulse was $2.2 \mathrm{~ns}$ long with about a 1 ns foot at $\sim 0.6-1$ TW per beam, followed by a $1 \mathrm{~ns}$ high power section which peaks at about 1.7 TW per beam. A typical laser pulse is shown in Fig. 1. For the drive experiments all ten Nova beams were used for a peak irradiation intensity of 17 TW. For the hot spot imaging experiments, only eight or nine of Nova's ten beams were used to avoid hitting the viewing slot. No measurable effect in the spot position was observed for a variation in total power of $\pm 11 \% \mathrm{rms}$. 
Scattering losses from stimulated Brillouin scattering (SBS) and stimulated Raman scattering (SRS) were measured on one (BL7) of the Nova beams. The scattered power and time-resolved spectra from both SBS and SRS scattered into the lens was measured using the full aperture backscatter station (FABS).[8] Scattering in the near back direction up to angles of $22^{\circ}$ was measured using the near backscatter imager (NBI).[16]

The geometry for the hot spot imaging experiments is shown in Fig. 2. A slot (typically $600 \mu \mathrm{m}$ high $\times 1900 \mu \mathrm{m}$ long) is cut along the length of the hohlraum in order to image the thermal $\mathrm{x}$-ray emission from heated $\mathrm{Au}$ walls. All hohlraums had $0.35 \mu \mathrm{m}$ polyimide windows covering the slot. The $\mathrm{CH}_{4}$-filled hohlraums also had $0.35 \mu \mathrm{m}$ polyimide across the laser entrance holes and were pressurized to $1 \mathrm{~atm}$. The slot had a fiducial wire extending about $100 \mu \mathrm{m}$ above its lower edge for absolute spatial position.

$X$ rays were imaged through the slot using two gated pinhole cameras (SXRFC) with $100 \mathrm{ps}$ temporal and $30 \mu \mathrm{m}$ spatial resolution.[17] For each shot, the SXRFC recorded four images separated in time by $\sim 0.5 \mathrm{~ns}$ for three energy bands at $\sim 280 \mathrm{eV}$, at $\sim 700 \mathrm{eV}$, and $>2 \mathrm{keV}$. One SXRFC viewed normal to the hohlraum axis $\left(0^{\circ}\right)$ and one viewed at $22^{\circ}$ to the normal. Diagnostic views and a typical pair of images at 0 and $22^{\circ}$ are shown in Fig. 3. Axial emission profiles are obtained by averaging across a $100 \mu \mathrm{m}$ wide strip near the middle of the slot (but away from the shadow of the fiducial wire). Continuous timeresolved one-dimensional images at $\sim 750 \mathrm{eV}$ were recorded using a soft $\mathrm{x}$-ray imager (SXI)[18] viewing from a $22^{\circ}$ line of sight. 
III. Thermal x-ray imaging

The geometry shown in Fig. 2 is used to measure $x$-ray emission profiles from the hohlraum wall. Previous experiments imaging $x$-ray emission profiles in vacuum hohlraums have shown that hot spot motion is consistent with modeling.[19, 20,21$]$ Emission profiles from the $22^{\circ}$ view at $700 \mathrm{eV}$ in the foot of the pulse (B) and near the peak of the pulse (A), are shown in Fig. 4 for $\mathrm{CH}_{4}$-filled and vacuum hohlraums. The emission profiles are averaged over at least two shots in all cases and as many as five shots for some of the data. The relative intensity profiles typically repeated to $10 \%$ after normalizing to the total intensity of the profile. The spatial profiles are fit with a Gaussian curve and a linear background to determine the center of gravity for hot spot emission. Although the spot may have significant structure, especially early in time as seen from Fig 4, this provides a convenient characterization of the emission distribution. The drive symmetry is determined by the entire emission profile. The resulting spot positions for each shot before averaging the profiles are shown in Fig. 5 for vacuum (open square) and $\mathrm{CH}_{4}$-filled (open circle) hohlraums for the $0^{\circ}$ view. The uncertainty in absolute position is about $\pm 40 \mu \mathrm{m}$ under nominally the same conditions. The average of several images within \pm 150 ps is shown in bold and the error bars, typically $\pm 20 \mu \mathrm{m}$, represent the error of the mean.

The experiments are modeled using 2- $d$ Lasnex.[22,23] The Nova beams are modeled as a cylindrically symmetric ring of laser light with approximately the same spatial extent of each Nova beam along the hohlraum axis. The incident power is the total laser power corrected for SBS and SRS scattering losses measured on a single beam.[24] Results of the modeling are post- 
processed to produce emission profiles similar to the experiments. Positions of the hot spot as a function of time are extracted from the simulations and are also plotted in Fig. 5. The uncertainty in the calculated spot positions is about $\pm 40 \mu \mathrm{m}$. The calculations do not predict a significant difference in the thermal emission distribution or hot spot position between $\mathrm{CH}_{4}$-filled and vacuum hohlraums. This is consistent with the predictions of timeintegrated symmetry where little difference was predicted between the pointing for best symmetry between $\mathrm{CH}_{4}$-filled and vacuum hohlraums. While the presence of gas modifies the evolution of the low-density plasma ablated by the laser, it is not predicted to significantly alter the position of $x$ ray emission for these experimental parameters.

At early times ( $\leq 500 \mathrm{ps}$ ), the measured hot spot center of gravity is displaced $60 \pm 25 \mu \mathrm{m}$ outward toward the LEH from the predicted position for $\mathrm{CH}_{4}$-filled hohlraums. The origin of this displacement can be seen in Fig. 4B by comparing the lineouts from $\mathrm{CH}_{4}$-filled and vacuum hohlraums since calculationally they are similar. For the vacuum hohlraum, the hot spot emission shows two distinct lobes with a hole in the center due to the structure in the Nova beam.[25] For the $\mathrm{CH}_{4}$-filled hohlraum, the intensity of the inner portion of the beam is depleted compared to the outer portion of the beam. The hole in the beam enhances the effect. In contrast, the intensity of the inner and outer portion of the beam is more nearly the same for the vacuum hohlraum. The inner ray of the beam has about twice the path length from the LEH to the wall compared to the outer ray, resulting in increased absorption of the inner portion of the beam in the underdense plasma. This effect leads to a small change in the hot spot center of gravity at early time in simulations of gas-filled hohlraums as shown in Fig. 5. The 
quantitative difference between the measured and calculated spot position at early time may be due to larger parametric losses along the longer path of the inner portion of the beam, or to increased absorption if the underdense plasma temperature is lower than predicted by modeling.

At later times (> $1 \mathrm{~ns}$ ), the hot spot center of gravity is similar for $\mathrm{CH}_{4}$-filled and vacuum hohlraums and agrees with calculations as shown in Fig. 5. The beam structure is not observed in Fig. $4 \mathrm{~A}$ because of thermal smoothing in the plasma. The complete emission profile outside of the hot spot, however, differs for $\mathrm{CH}_{4}$-filled hohlraums compared with vacuum hohlraums which significantly affects symmetry. The emission intensity outside of the spot from the "thermally" heated plasma is increased by $~ 12 \pm 6 \%$ near the LEH. As discussed later, the increased intensity near the LEH is consistent with filamentation in a transverse flow.[12,13] The intensity near the midplane is less for a $\mathrm{CH}_{4}$-filled hohlraum due to larger scattering losses for $\mathrm{CH}_{4}$-filled hohlraums which reduces the drive.

We use a three-dimensional view factor code to estimate the effective shift in pointing of best symmetry due to changes in emission profiles in $\mathrm{CH}_{4}$-filled hohlraums. The changes are equivalent to a $\sim 100 \mu \mathrm{m}$ outward pointing shift. This is slightly less than the $130 \mu \mathrm{m}$ outward shift reported in Ref. 11 but within the errors of the experiments and calculations. This correlation between the $x$-ray emission pattern and the shift in symmetry confirms that the capsules in $\mathrm{CH}_{4}$-filled hohlraums are driven by $\mathrm{x}$ rays and not by other non-thermal sources. 
The effects of beam smoothing on the thermal emission profile are tested by placing an RPP in one of the Nova beams. The RPP produces an Airy pattern in the far field intensity distribution envelope with $\sim 83 \%$ of the intensity in the central spot.[26] The central maximum for the Nova RPP is $\sim 700 \mu \mathrm{m} x$ $370 \mu \mathrm{m}$ in the far field which is placed in the LEH.[27] This produces a nearly circular spot of $\sim 700 \mu \mathrm{m}$ dia. on the hohlraum wall. Within this envelope, the RPP produces a speckle pattern of hot spots with transverse size, $\delta x=\pi \lambda f$, where $\lambda$ is the laser wavelength, $f$ is the f-number of the lens. For Nova $f \sim 4.3$ and $\lambda=0.35 \mu \mathrm{m}$, so $\delta \mathrm{x} \sim 4 \mu \mathrm{m}$. In contrast, a typical Nova beam has a significantly higher fraction of its power at higher intensity in phaseaberration-produced hot spots of larger scale.

The RPP should reduce effects of filamentation compared to an unsmoothed Nova beam. A comparison of the intensity distribution of a typical Nova beam and an RPP beam at the plane of the LEH is shown in Fig. 6. Plotted is the fraction of the beam above a given intensity for a beam with 2 TW of power. These estimates are derived from the Nova beam model developed in ref. 25 and modification by the RPP developed in ref. 27. For plasma conditions calculated at the $\mathrm{LEH}$, filamentation thresholds are estimated to be $\sim 6 \times 10^{15} \mathrm{~W} / \mathrm{cm}^{2}$. For a typical Nova beam $\sim 50 \%$ of the intensity is greater than $6 \times 10^{15} \mathrm{~W} / \mathrm{cm}^{2}$ while less than $20 \%$ of an RPP beam is greater than that intensity. In addition, the RPP increases the length over which the beam maintains the far field distribution so that the beam is still nearly in the far field when it reaches the hohlraum wall.

Lineouts from the RPP beam are shown at $t \sim 0.9 \mathrm{~ns}$ and near the peak of the pulse ( $t$ 1.3 ns) in Fig. 7B and 7A, respectively, for $\mathrm{CH}_{4}$-filled and vacuum 
hohlraums. The center of gravity of the hot spots extracted from the lineouts are shown in Fig. 5. No significant difference in the spot position is observed with and without an RPP for vacuum hohlraums. However, the addition of an RPP significantly changes the hot spot position for $\mathrm{CH}_{4}$-filled hohlraums. The difference between experiments and calculations of the hot spot center of gravity is eliminated for $t \geq 0.5 \mathrm{~ns}$ (we have no data before that time). In addition, at the peak of the pulse the enhanced emission near the LEH is eliminated.

At early times, changes in the RPP intensity distribution envelope contribute significantly to the reduction in the observed shift. The RPP produces a single spot on the wall since it is still approximately in the far field instead of an annulus which produces two lobes when imaged through the center. The path length difference between the inner ray and outer ray is much less for the RPP compared to a regular Nova beam reducing effects of differential absorption across the beam spot. The lower intensity distribution for the RPP helps to suppress any possible stimulated processes which might also be contributing to the larger losses in the inner portion of a regular Nova beam.

Near peak intensity the RPP suppresses the enhanced thermal emission near the $\mathrm{LEH}$ for $\mathrm{CH}_{4}$-filled hohlraums. This is consistent with the interpretation that the enhanced emission results from beam deflection in a plasma with a transverse flow gradient. The calculated deflection of the beam for a hot spot with an intensity of about $6 \times 10^{15} \mathrm{~W} / \mathrm{cm}^{2}$ is $\sim 9^{\circ}$.[13] This angle is consistent with enhanced emission toward the LEH observed in the experiment for unsmoothed beams since they have significantly more structure at higher intensities than an RPP beam. Simulations show that reducing the intensity 
of the hot spot reduces the deflection, consistent with the observation that an RPP reduces the excess emission near the LEH.

Spatial beam smoothing also reduces levels of SBS and SRS. In single beam experiments, an RPP and a kinoform phase plate (KPP) are used in BL7 which has the backscatter diagnostic.[28] A KPP is similar to an RPP in that it reduces the fraction of the beam energy at high intensity as shown in Fig. 6 . The advantage of a KPP is that it can produce a more uniform average intensity in the central spot. The present KPP produces an elliptical far field spot approximately $220 \mu \mathrm{m}$ by $340 \mu \mathrm{m}$ containing $~ 93 \%$ of the incident laser energy. Time integrated SBS and SRS scattering into the lens is shown in Fig. 8. The figure compares scattering levels with and without a KPP in a single beam. The RPP and KPP reduces the total scattering to less than $3 \%$ compared to nearly $15 \%$ without a KPP.

IV. Ten smoothed beam results

The encouraging results from single beam experiments provided impetus to field beam smoothing on all ten of Nova's beams to perform integrated experiments on $x$-ray drive and symmetry. Ten KPP's were fabricated with each producing an elliptical spot at best focus that is $220 \mu \mathrm{m}$ by $340 \mu \mathrm{m}$. The elliptical design was chosen to produce an approximately circular spot in the plane of the laser entrance hole with $2.2 \AA$ of SSD. The average intensity in the beam for a $2 \mathrm{TW}$ beam is $\sim 3 \times 10^{15} \mathrm{~W} / \mathrm{cm}^{2}$ at best focus which is slightly higher than the average intensity of a NIF beam at the LEH which is $-2 \times 10^{15} \mathrm{~W} / \mathrm{cm}^{2}$. 
$X$-ray images of five of the Nova beams are shown in Fig. 9 for beams with and without a KPP. The beams did not have SSD on them for these experiments. The unsmoothed Nova beams have been defocused by $1 \mathrm{~mm}$ corresponding to the focal conditions at the plane of the laser entrance hole. For the beams without KPP's there is considerable structure due to phase aberrations from the amplifiers, the split in the disk of the $46 \mathrm{~cm}$ dia. amplifiers, and from the support structure of the KDP array.[25]. The hole in the center of the beam is due to the center $20 \mathrm{~cm}$ beam block in the Nova beam so that unconverted light will not hit the target and that internal ghost foci in the final focus lens are reduced. The KPP beams show much less structure although they are not perfectly uniform because of residual beam phase front aberrations. The KPP beam has the small scale speckle pattern characteristic of the $f / \#$ of the lens. Beam statistics for a KPP is compared in Fig. 6 with a normal Nova beam and an RPP beam. The KPP beam has slightly more energy at higher intensity than an RPP beam primarily because a KPP beam has a more uniform distribution and more energy in the central maximum.

The $\mathrm{x}$-ray drive in $\mathrm{CH}_{4}$-filled hohlraums with ten KPP beams increased significantly compared to the drive from $\mathrm{CH}_{4}$-filled hohlraums heated with unsmoothed Nova beams. A comparison of drive temperature is shown in Fig. 10 for both vacuum and $\mathrm{CH}_{4}$-filled hohlraums without beam smoothing, with KPP's alone, and with KPP's plus $2.2 \AA$ of SSD. The data are the measured Dante wall temperature at $1.8 \mathrm{~ns}$ near the peak of the drive pulse corrected by $11 \mathrm{eV}$ for the wall albedo.[4] For $\mathrm{CH}_{4}$-filled hohlraums the drive increases by $\sim 15 \mathrm{eV}$ with the addition of KPP's and KPP's+SSD to temperatures above $210 \mathrm{eV}$. The drive from vacuum hohlraums also 
increases with KPP's due to reduced scattering losses. In other experiments using a pulse with peak intensity of $\sim 25 \mathrm{TW}$, peak drive temperatures of $230 \mathrm{eV}$ have been obtained in $\mathrm{CH}_{4}$-filled hohlraums.

The increased drive temperature is due mostly to increased laser coupling to the target. Fig. 11 shows the absorption fraction for the different beam configurations. For targets with unsmoothed Nova beams, absorption is 80 $85 \%$ of the incident laser energy. SBS is $9-12 \%$ and SRS is $6-8 \%$. When KPP's are added, absorption is greater than $90 \%$ with the SRS being reduced to less than $2 \%$ and the SBS to $~ 5 \%$. With $2.2 \AA$ SSD, absorption is greater than $95 \%$ and the SBS and SRS is approximately halved compared to KPP's only. The time history of the incident laser power and the scattering losses is shown in Fig. 11 for KPP's + SSD. The scattering occurs around the peak of the pulse , but the peak absorbed power is still more than $90 \%$ of the incident peak power.

The KPP's reduce the fraction of energy above $6 \times 10^{15} \mathrm{~W} / \mathrm{cm}^{2}$, the filamentation threshold for conditions near the laser entrance hole, compared to a Nova beam. This improves target performance in two important ways. First, it reduces the levels of SRS from the underdense plasma. The SRS spectrum from an unsmoothed beam is quite broad and shifted to shorter wavelengths. This infers that much of the SRS is occurring in the density depression produced by the filament. Most of the SRS from low densities is eliminated with KPP's accounting for the reduction in SRS levels.

The second improvement is the reduction in beam deflection since beam deflection occurs from filamentation in a transverse flow near the expanding 
plasma sonic point. In symmetry experiments reported elsewhere, the shift in symmetry for gas-filled hohlraums is significantly reduced with KPP's on all ten beams.[14] Further evidence can be seen from the angular distribution of SBS. SBS is produced in the plasma $\sim 0.1 n_{c}$ near the hohlraum wall. If the incident beam is deflected traversing the sonic point in the underdense plasma before reaching the wall, SBS at the wall would be shifted from direct backscatter by the deflection angle. For unsmoothed Nova beams the SBS is shifted by about $9^{\circ}$ from direct backscatter consistent with beam deflection. Over $50 \%$ of the SBS light is scattered outside of the lens. With a KPP beam, the SBS is nearly collimated and only $\sim 20 \%$ of the SBS light is scattered outside of the lens.

\section{Summary}

In summary, both single beam and ten beam experiments have shown that beam smoothing significantly improves gas-filled hohlraum target performance. With unsmoothed Nova beams, total scattering levels from SBS and SRS are $15-20 \%$ and the pointing of beast symmetry is shifted by $\sim 130 \mu \mathrm{m}$ compared to Lasnex modeling predictions. Single beam experiments show that much of the pointing shift is due to enhanced heating near the endcap of the hohlraum. Some of the effect is due to early time beam propagation through the low $\mathrm{Z}$ gas. The heating of the endcap is consistent with filamentation of the beam in a transverse flow. Both effects can be reduced using spatial beam smoothing. In addition spatial beam smoothing reduced the scattering levels from SBS and SRS. Experiments with smoothing on all ten beams confirm the single beam results. Drive measurements with KPP's and KPP's+SSD showed that drive increased by $\sim 15 \mathrm{eV}$ in gas-filled 
hohlraums compared to drive in gas-filled hohlraums heated with unsmoothed beams. Peak drive temperatures are measured to be $210 \mathrm{eV}$ for 17 TW peak power pulses and $230 \mathrm{eV}$ for pulses with peak power of $25 \mathrm{TW}$. Scattered light measurements show that absorption is over $95 \%$ for gas-filled hohlraums using KPP's with $2.2 \AA$ of SSD. In symmetry experiments, reported elsewhere, the shift in pointing of best symmetry is reduced to $35 \mu \mathrm{m}$ from the best symmetry position predicted by Lasnex which is nearly the pointing and alignment accuracy of Nova.[14] These results using beam smoothing being planned for NIF provide confidence in target performance predictions for NIF.

We would like to thank the support of the Nova personnel for operations of Nova and to LANL technical staff for support of the gated $x$-ray imagers. We also acknowledge contributions of Norm Delamater and other LANL scientists for sharing the results of the time integrated symmetry experiments prior to publication. This work supported under the auspices of the U.S. Department of Energy by the Lawrence Livermore National Laboratory under Contract No. W-7405-ENG-48.

1 . J. Lindl, Phys. Plasmas 2, 3933 (1995).

2. S. W. Haan et al., Phys. Plasmas 2, 2480 (1995).

3 . E. M. Campbell, et al., Rev. Sci. Instrum. 57, 2101 (1986).

4 R. L. Kauffman, et al., Phys. Rev. Lett. 73, 2320 (1994).

5 . L. J. Suter, et al., Phys. Plasmas 3, 2057 (1996).

6. L. J. Suter, et al., Phys. Rev. Lett. 73, 2328 (1994). 
7. A. A. Hauer, et al., Phys. Plasmas 2, 2488 (1995).

8 . B. J. MacGowan et al., P. Plasmas 3, 2029 (1996)

9. J. D. Fernandez, et al., Phys. Rev. Lett., 77, 2702 (1996).

10. T. J. Orzechowski, private communication

11 . N. D. Delamater, et al., Phys. Plasmas 3, 2022 (1996).

12. H. Rose, Phys. Plasmas 3, 1709 (1996).

13 . D. Hinkel, E. A. Williams, and C. H. Still, Phys. Rev. Lett. 77, 1298 (1996).

14 N. D. Delamater, to be published.

15 H. N. Kornblum, R. L. Kauffman, and J. A. Smith, Rev. Sci. Instrum. 57, 2179 (1986).

16 R. K. Kirkwood et al., Rev. Sci. Instrum. 68, 636 (1997)

17 . F. Ze et al., Rev. Sci. Intsrum. 63, 5124 (1992).

18 . R. Sigel et al., Phys. Rev. A 45, 3987 (1992); also, J. Porter, private communication (1994).

19 . F. Ze, private communication.

20 . D. B. Ress, et al., Bull. Am. Phys. Soc. 38, 1885 (1993).

21. L. V. Powers, et al., Defense Research Review 6-10, (1994).

22. G. Zimmerman and W. L. Kruer, Comments Plasma Phys. Controlled Fusion 2, 51 (1975).

23 . L. V. Powers, et al., submitted to Phys. Plasmas.

24 . B. J. MacGowan, et al., Phys. Plasmas 3, 2029 (1996). 
25. P. Wegner, Report No. UCRL-ID-110480, Vol. 2, 1992.

26. S. Skupsky, et al., J. Appl. Phys. 66, 3456 (1989)

27. S. N. Dixit et al., Applied Optics 32, 2543 (1993).

28 . S. N. Dixit et al., Optics Lett. 21, 1715, (1996). 
Figure captions.

Figure 1. Time dependence of laser power is shown as a function of time. Most data are taken with peak powers of 17-20 TW and pulses having contrasts of 3:1 to 2:1.

Figure 2. Geometry of the diagnostic view of the $x$-ray spot. As the gold plasma moves away from the wall, the position of the peak emission changes.

Figure 3. X-ray images, superposed on the target photographs, of laser-plasma spots inside the methane-filled hohlraums, from $22^{\circ}$ (top, $700 \mathrm{eV}$ channel) and $0^{\circ}$ (bottom, $>2 \mathrm{keV}$ channel). The images are 1.3 and $0.5 \mathrm{~ns}$ after the start of the laser pulse.

Figure 4. Axial emission profile at $700 \mathrm{eV}$ as viewed from the $22^{\circ}$ view, for $\mathrm{t}=$ $0.9 \mathrm{~ns}(\mathrm{~B})$ and $1.3 \mathrm{~ns}(\mathrm{~A})$ for a typical Nova beam. The vacuum hohlraum is shown by a solid line and the methane-filled by a dashed line. The emission decreases at $1500 \mu \mathrm{m}$ from the midplane due to the laser entrance hole.

Figure 5. Spot position vs. time for a beam with and without an RPP, viewed from $0^{\circ}$. The calculated positions are the lines and the SXRFC data are the markers. The data without an RPP are shown as open circles (methane-filled hohlraums) and open squares (vacuum hohlraums), and the data with an RPP are filled circles (methane) and squares (vacuum). The bold markers are the data averaged over 150 ps intervals. 
Figure 6. The fraction of beam energy above a given intensity versus intensity. The intensity distribution is shown for a normal Nova beam, a Nova beam with a random phase plate (RPP), and a Nova beam with a kinoform phase plate (KPP).

Figure 7. Axial emission profile at $700 \mathrm{eV}$ as viewed from the $22^{\circ}$ view, for $\mathrm{t}=$ $0.9 \mathrm{~ns}$ (B) and $1.3 \mathrm{~ns}$ (A) for an RPP beam. The vacuum hohlraum is shown by a solid line and the methane-filled by a dashed line.

Fig. 8. Measured scattering levels from a single Nova beam. Time integrated SBS and SRS scattering fractions are shown for a Nova beam without beam conditioning and a Nova beam with a KPP and RPP.

Fig. 9. X-ray images with energy $>2 \mathrm{keV}$ for five Nova beams. The image on the left is of standard Nova beams without beam conditioning at $2 \mathrm{~mm}$ past best focus. The image on the right is with all beams having KPP's. The images are taken at approximately the position of the hohlraum wall.

Fig. 10. X-ray drive from vacuum and $\mathrm{CH} 4$-filled hohlraums. Data are shown for irradiation without beam smoothing, with KPP's and with KPP's and $2.2 \AA$ SSD.

Fig. 11. Time history of the incident laser pulse and SBS and SRS scattering. The Absorbed laser power is derived from subtracting the SBS and SRS levels from the incident laser pulse. 


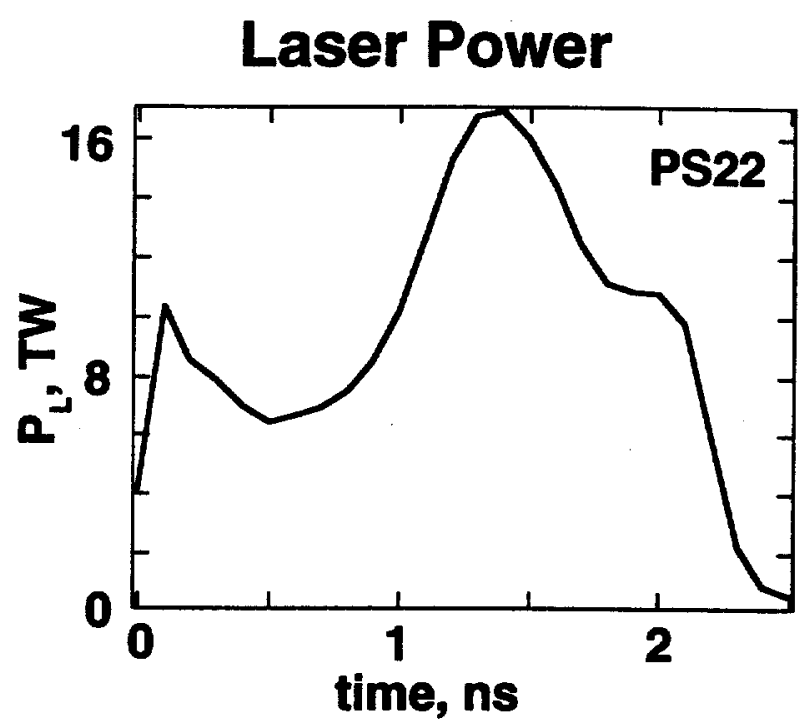

Figure 1 


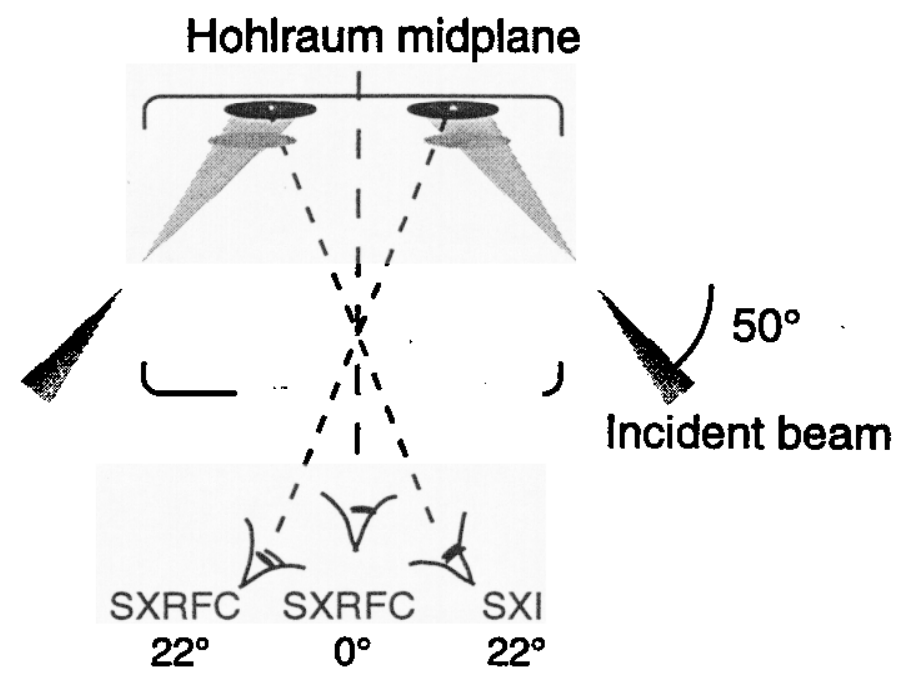

Figure 2 

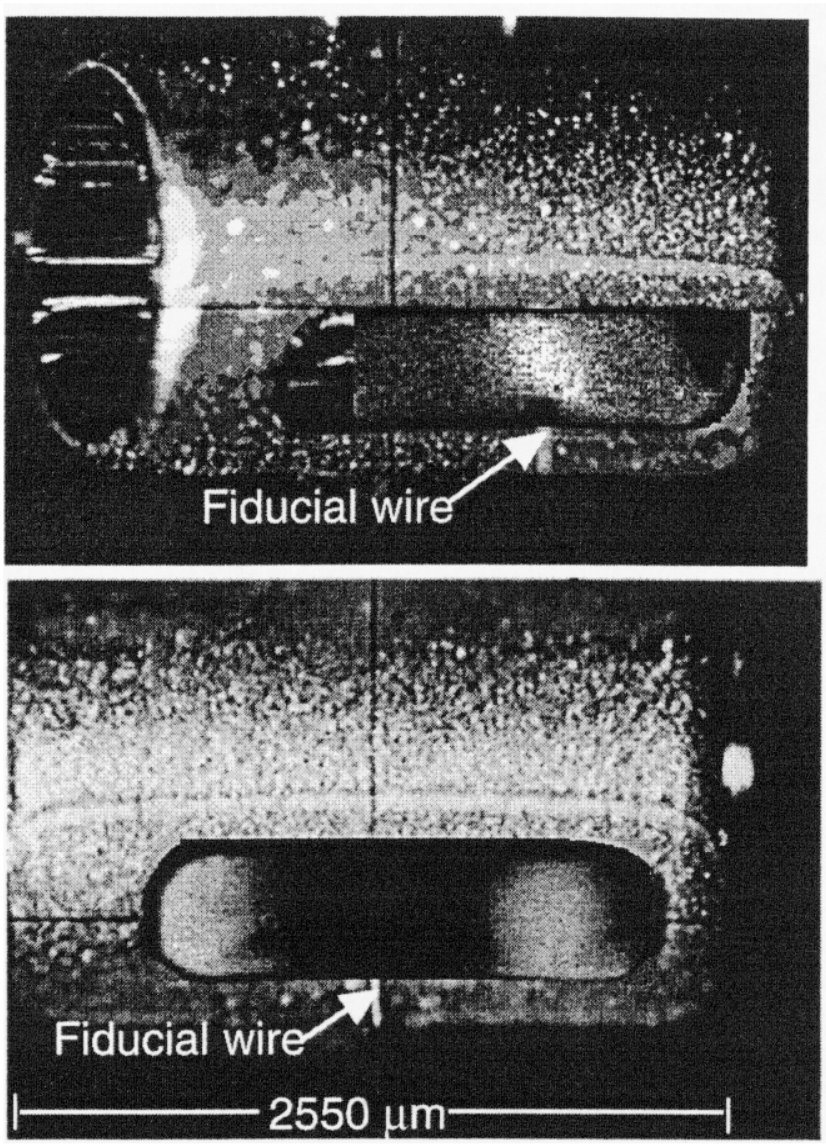

Figure 3 


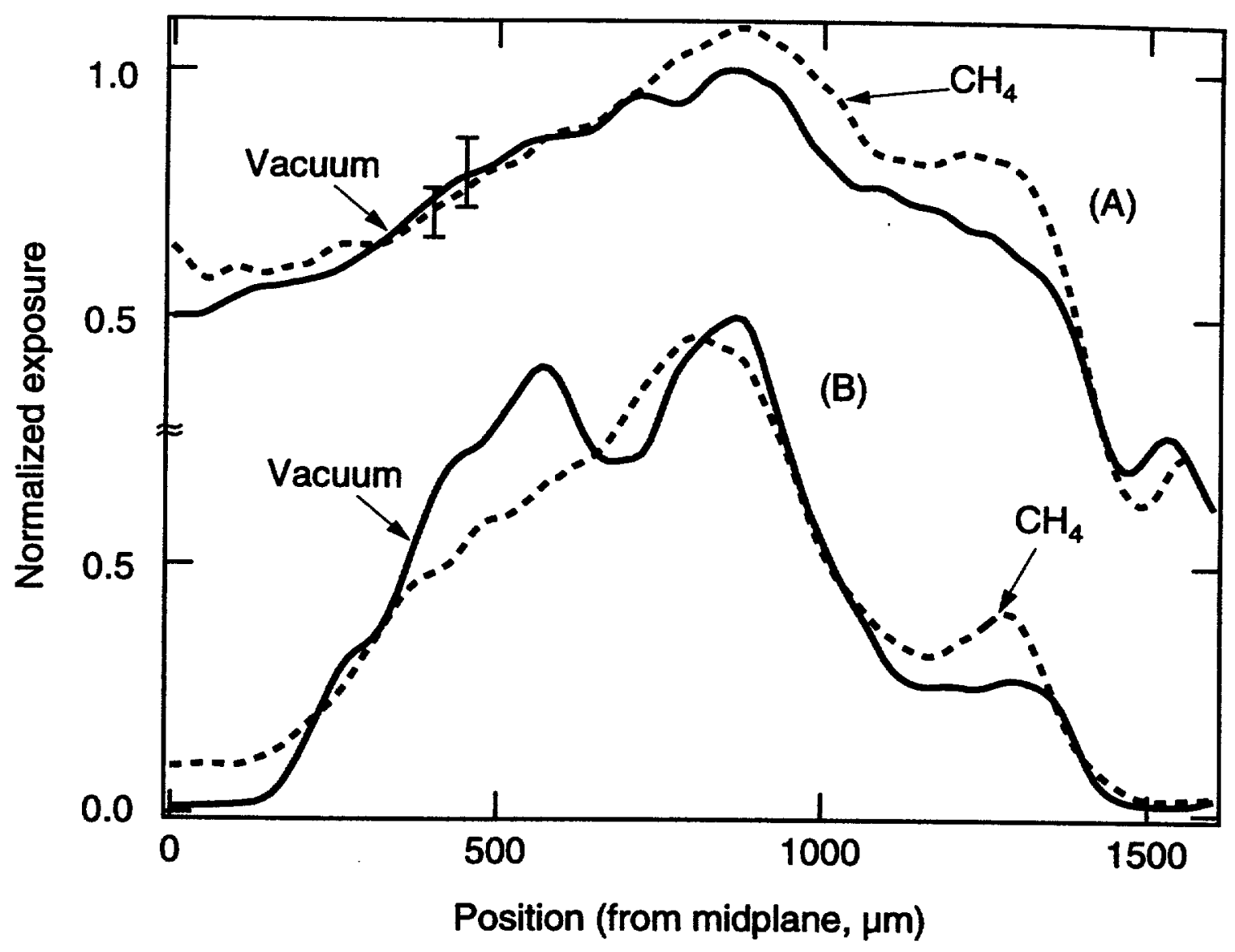

Figure 4 


\section{$\because$}

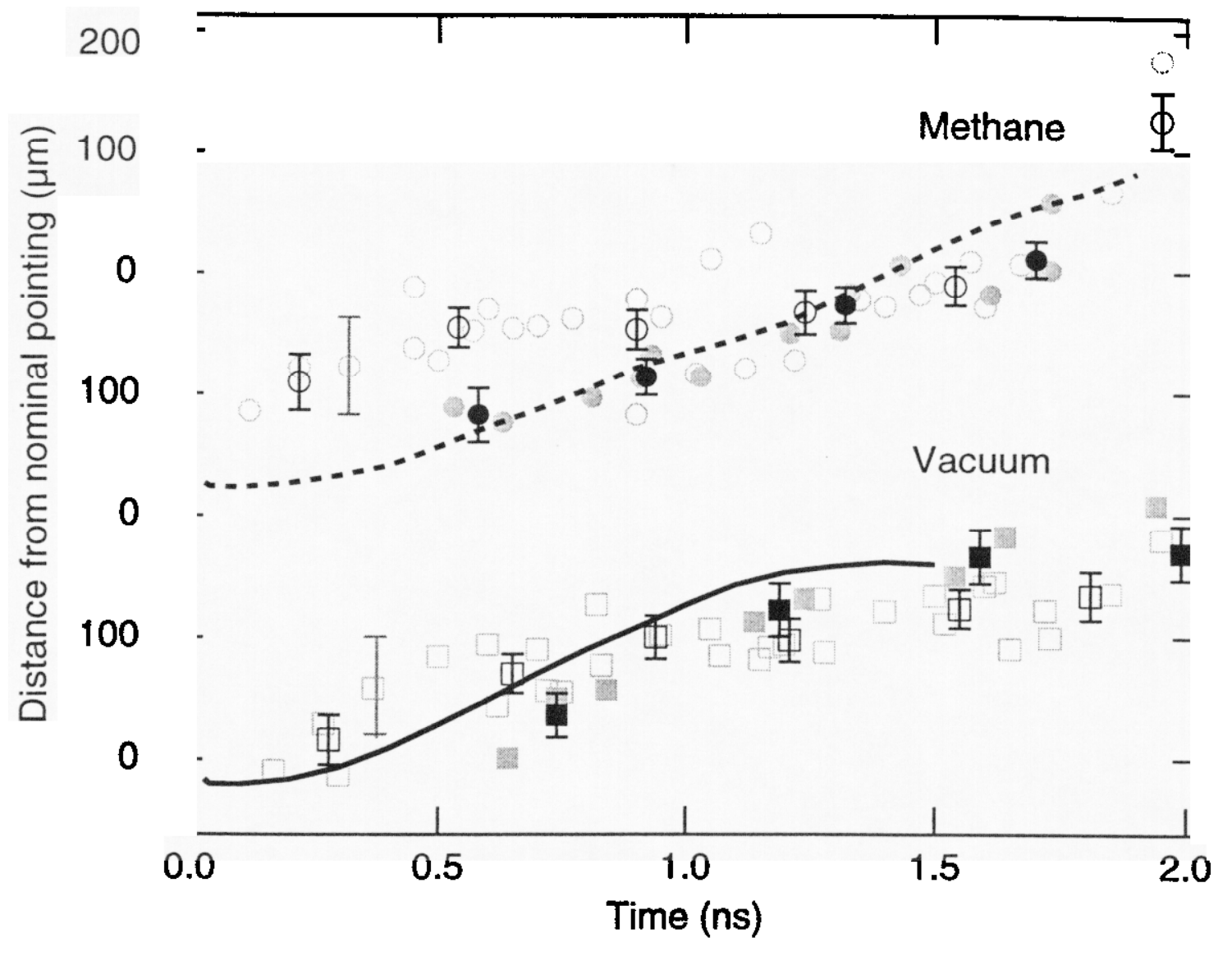

Figure 5 


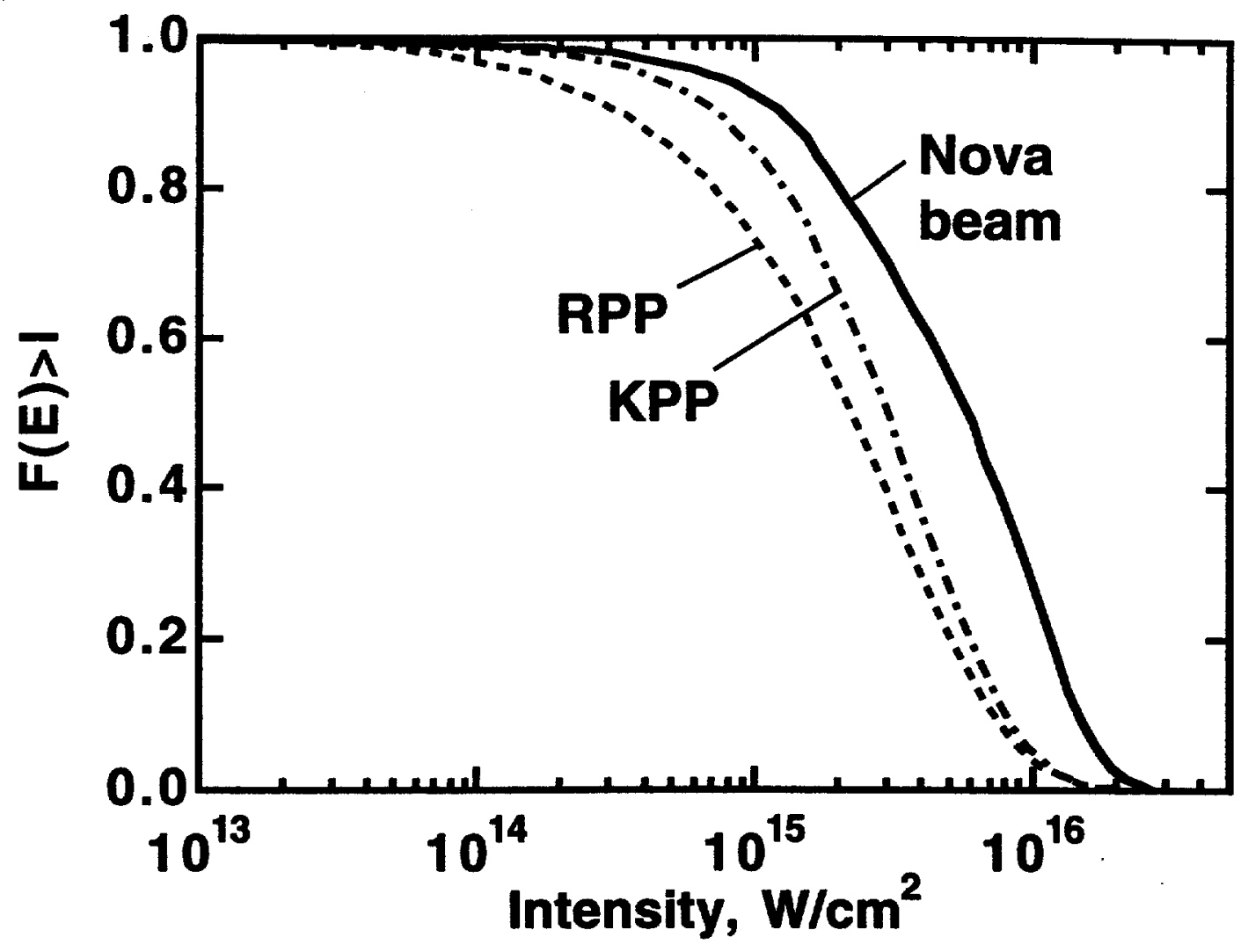

Figure 6 


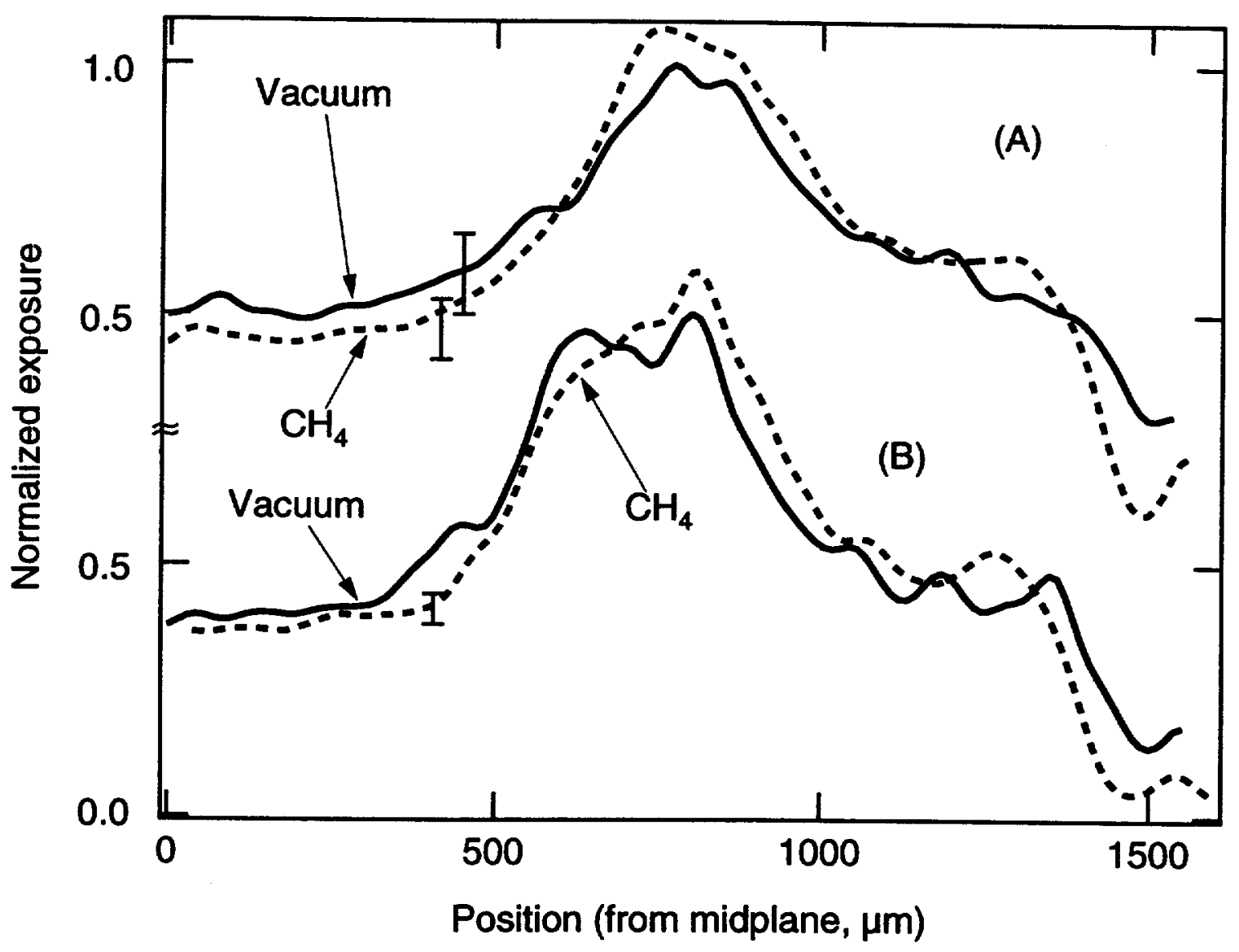

Figure 7 


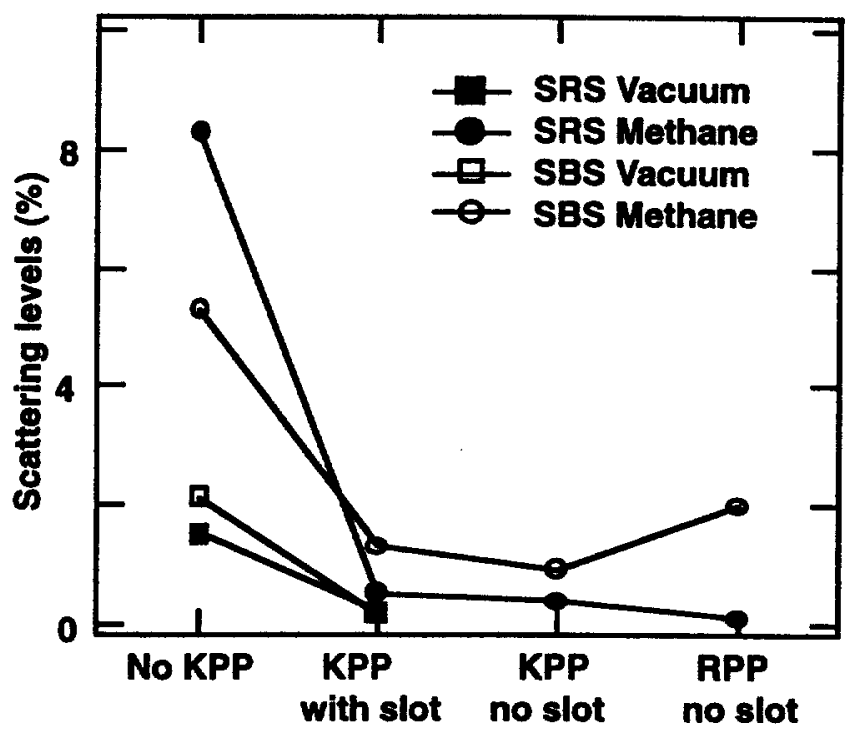

Figure 8 


\section{Kinoform phase plates (KPPs) produce}

\section{a much smoother beam on Nova}

\section{Before}

Unsmoothed (2-mm defocus)

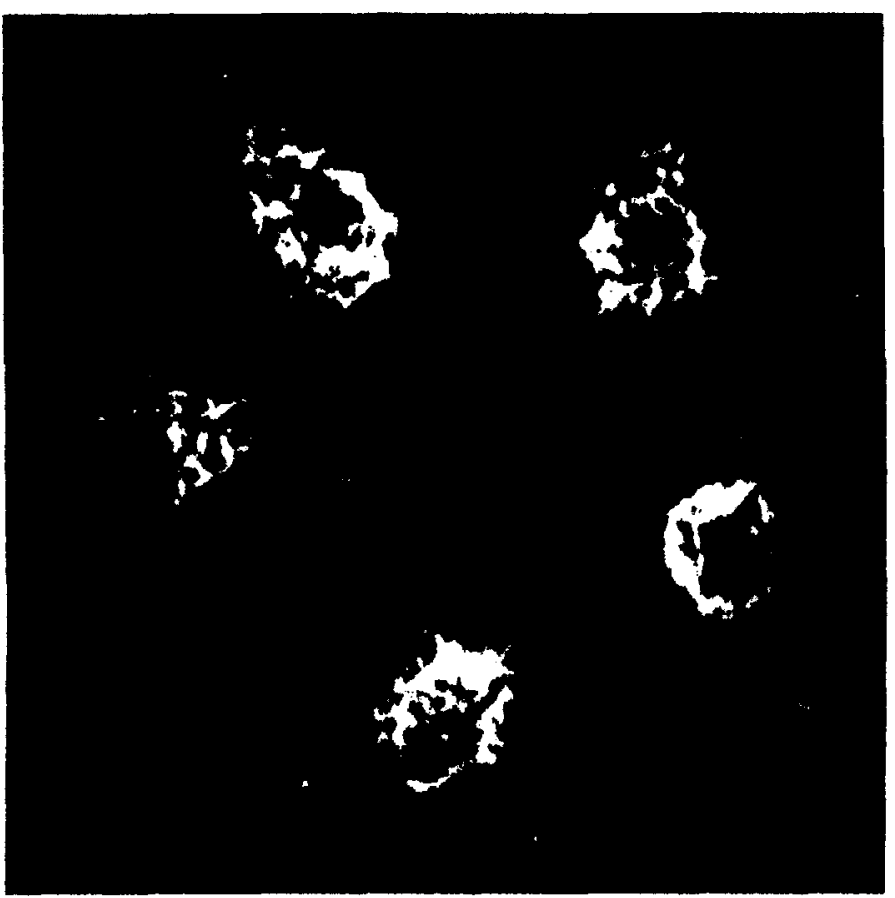

\section{After}

With KPP (1-mm defocus)

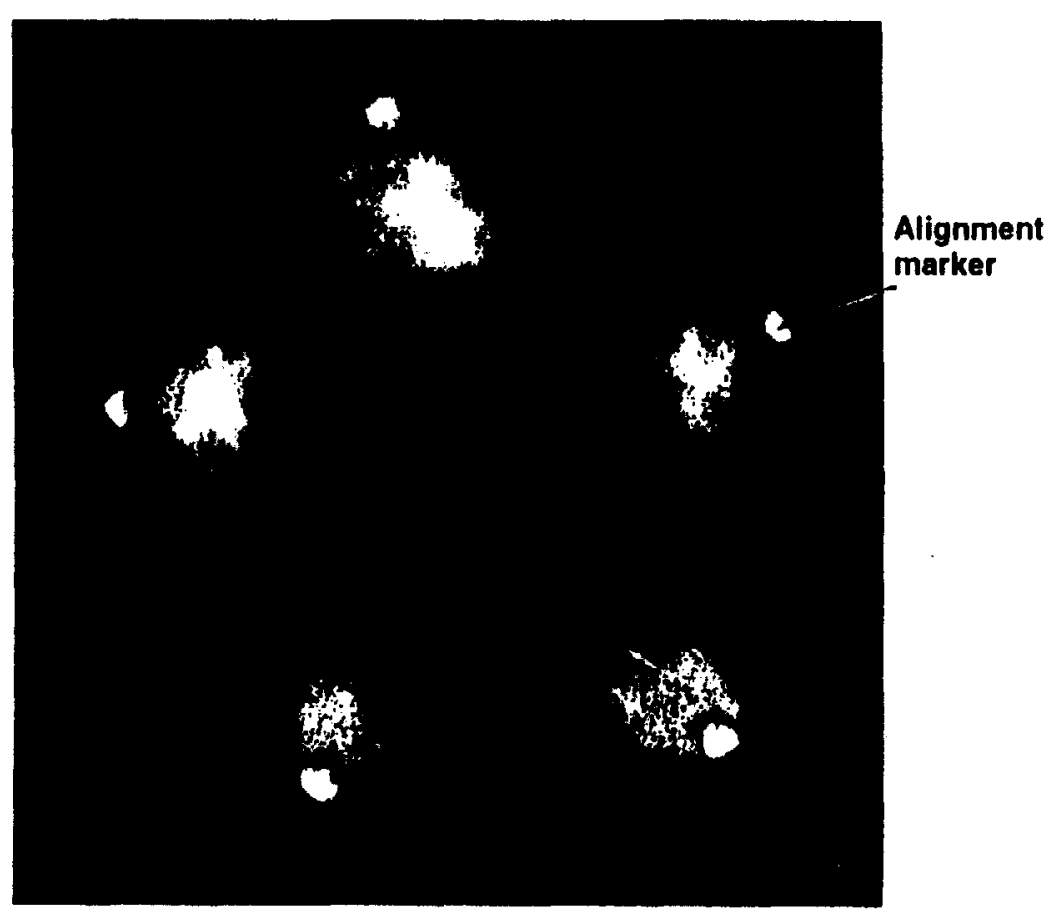

2 keV x-ray images 


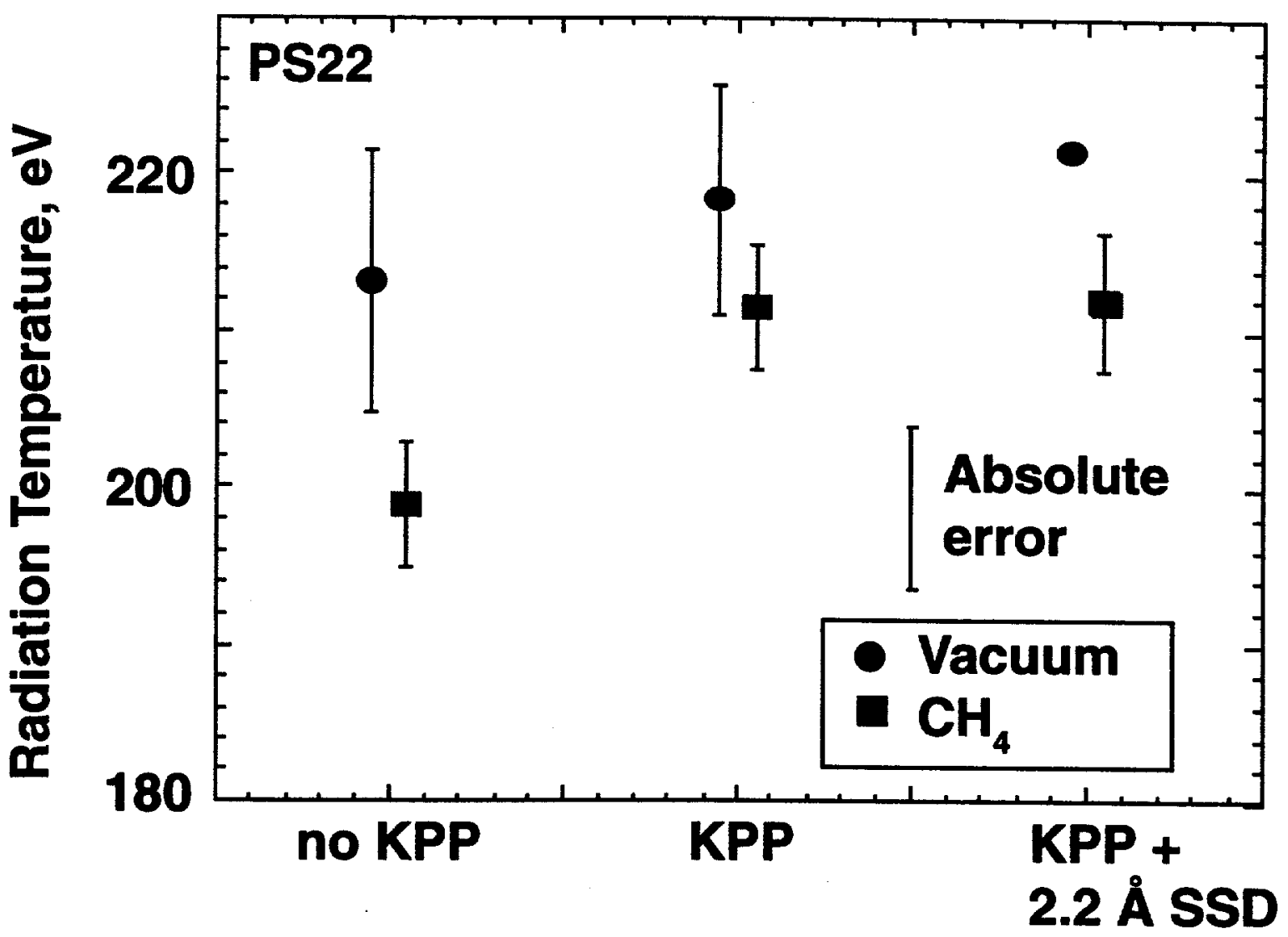

Figure 10 

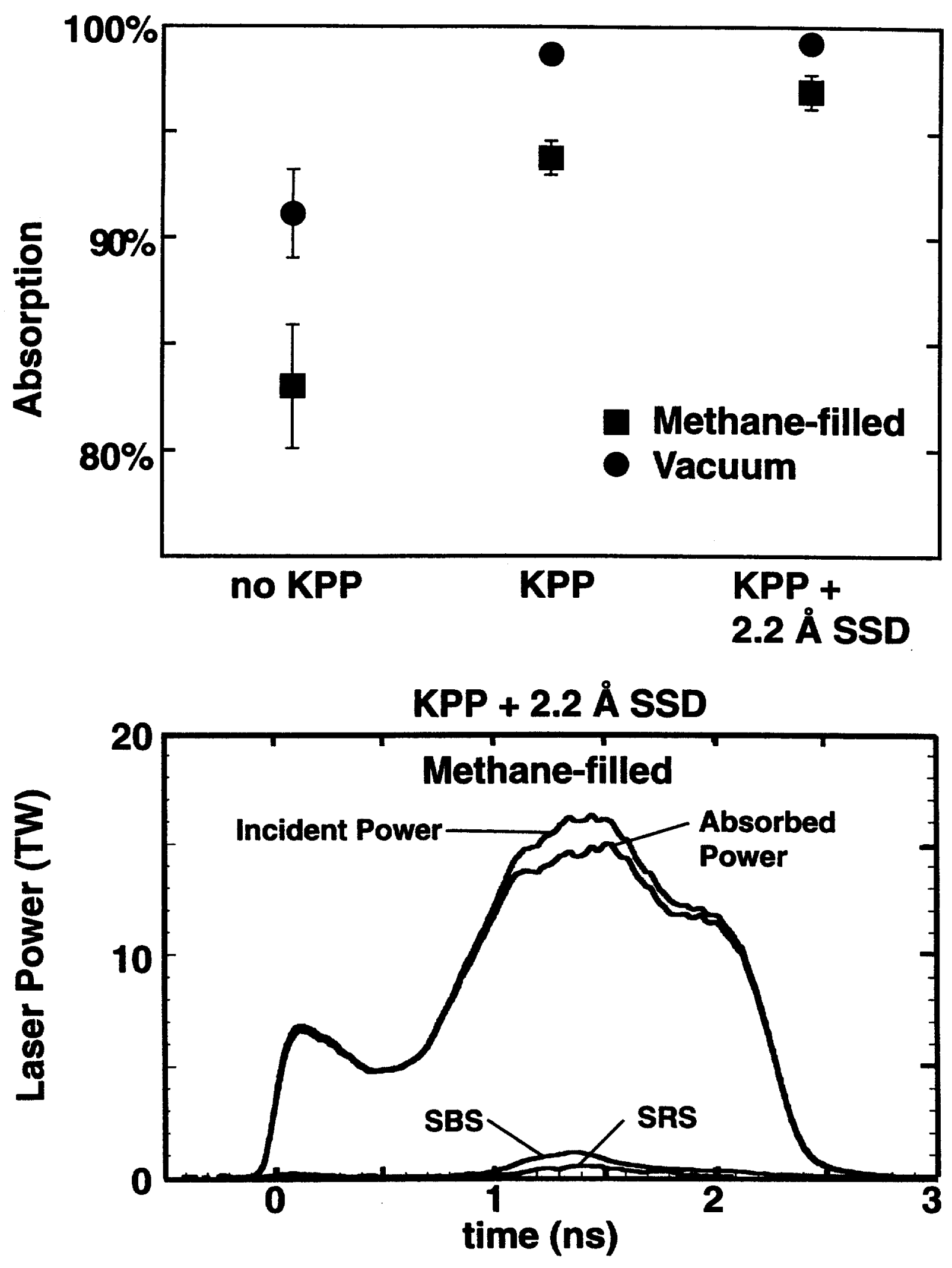

Figure 11 


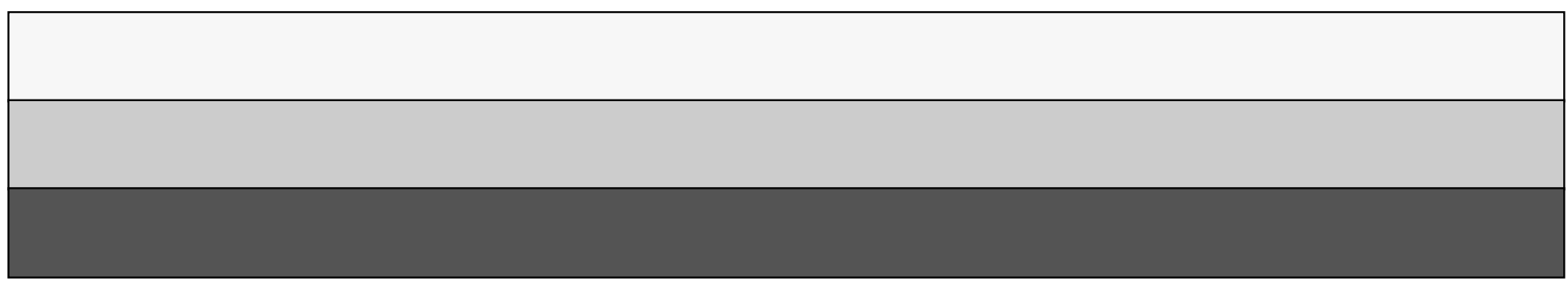

